\title{
MT1-MMP deficiency leads to defective ependymal cell maturation, impaired ciliogenesis, and hydrocephalus
}

\author{
Zhixin Jiang, ${ }^{1,2}$ Jin Zhou, ${ }^{1,2}$ Xin Qin, ${ }^{1,2}$ Huiling Zheng, ${ }^{1,3}$ Bo Gao, ${ }^{1}$ Xinguang Liu, ${ }^{3}$ Guoxiang Jin, ${ }^{4}$ \\ and Zhongjun Zhou ${ }^{1,2}$ \\ ${ }^{1}$ School of Biomedical Sciences, Li Ka Shing Faculty of Medicine, University of Hong Kong, Hong Kong, China. ${ }^{2}$ Shenzhen \\ Institute of Innovation and Research, University of Hong Kong, Shenzhen, China. ${ }^{3}$ Institute for Aging Research, \\ Guangdong Provincial Key Laboratory of Medical Molecular Diagnostics, Guangdong Medical University, Dongguan, China. \\ ${ }^{4}$ Guangdong Provincial People's Hospital, Guangdong Academy of Medical Sciences, Guangzhou, China.
}

\begin{abstract}
Hydrocephalus is characterized by abnormal accumulation of cerebrospinal fluid (CSF) in the ventricular cavity. The circulation of CSF in brain ventricles is controlled by the coordinated beating of motile cilia at the surface of ependymal cells (ECs). Here, we show that MT1-MMP is highly expressed in olfactory bulb, rostral migratory stream, and the ventricular system. Mice deficient for membrane-type 1-MMP (MT1-MMP) developed typical phenotypes observed in hydrocephalus, such as dome-shaped skulls, dilated ventricles, corpus callosum agenesis, and astrocyte hypertrophy, during the first 2 weeks of postnatal development. MT1-MMP-deficient mice exhibited reduced and disorganized motile cilia with the impaired maturation of ECs, leading to abnormal CSF flow. Consistent with the defects in motile cilia morphogenesis, the expression of promulticiliogenic genes was significantly decreased, with a concomitant hyperactivation of Notch signaling in the walls of lateral ventricles in $\mathrm{Mmp}^{14^{-/-}}$brains. Inhibition of Notch signaling by $\gamma$-secretase inhibitor restored ciliogenesis in Mmp14 ${ }^{-/-}$ECs. Taken together, these data suggest that MT1-MMP is required for ciliogenesis and EC maturation through suppression of Notch signaling during early brain development. Our findings indicate that MT1-MMP is critical for early brain development and loss of MT1-MMP activity gives rise to hydrocephalus.
\end{abstract}

Conflict of interest: The authors have declared that no conflict of interest exists.

Copyright: () 2020, American Society for Clinical Investigation.

Submitted: August 16, 2019

Accepted: March 26, 2020

Published: May 7, 2020.

Reference information: /CI Insight. 2020;5(9):e132782.

https://doi.org/10.1172/jici.

insight.132782.

\section{Introduction}

Hydrocephalus, a common birth defect with an estimated incidence of 1:1000 (1), is characterized by enlarged intracerebral ventricles. It causes convulsions, mental disability, and even death in patients (2). Hydrocephalus can result from either the outflow blockage of cerebrospinal fluid (CSF) in ventricles, or CSF overproduction, or the impaired absorption of CSF. The identification of genetic risk factors is fundamental to our understanding of the molecular mechanisms underlying the pathogenesis of hydrocephalus and serves as the key in the diagnosis and management $(3,4)$. Recent studies using genetically modified

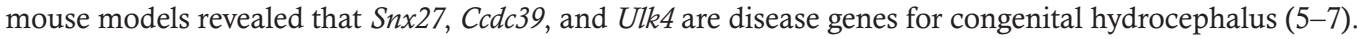

Ependymal cells (ECs) lining the cerebral ventricles are derived from the radial glia. They are fully differentiated by the second week of postnatal life. ECs are essential for the maintenance of brain homeostasis through the regulation of the neurogenic niche (8), establishing CSF flow (9), and forming a neuroprotective metabolic barrier over the ventricular surface (10). Defects in the generation, maturation, or integrity of ECs lead to early hydrocephalus. ECs are covered by motile cilia whose coordinated beating facilitates the directional flow of CSF. Therefore, defect in cilia is closely associated with hydrocephalus (11)

MMPs form a $\mathrm{Zn}^{2+}$-dependent proteinase superfamily, capable of degrading pericellular and extracellular proteins. In the CNS, MMPs have been implicated as involved in a variety of neurological disorders, such as Alzheimer's disease, Parkinson's disease, Multiple sclerosis, glioma invasion, and neuroinflammation (12). In experimental hydrocephalus, the elevated MMP9 and MMP7 were detected in CSF in adult rats after intraventricular Kaolin injection $(13,14)$. However, the role of MMPs in congenital hydrocephalus remains unknown.

Membrane-type 1-MMP (MT1-MMP), a cell membrane-bound MMP encoded by MMP14, is the most important and extensively investigated member of the MMP family. It plays important roles in diverse 
physiological and pathological conditions through pericellular proteolysis and extracellular remodeling (15). MT1-MMP-deficient mice exhibit dwarfism, skeletal abnormalities, and soft tissue defects and die before adulthood, suggesting a crucial function of MT1-MMP in development and postnatal growth (16-21). Recent studies proposed that MT1-MMP may be a potential therapeutic target in glioblastoma (22-24). However, the role of MT1-MMP in the developing brain remains largely unexplored.

In this study, we examined the developmental abnormalities in the ventricular system in Mmp14-deficient mice. We showed that MT1-MMP is highly expressed in EC linages lining the ventricles. MT1-MMPnull mice exhibited reduced and disorganized motile cilia that likely give rise to abnormal CSF flow. Consistent with this observation, loss of MT1-MMP resulted in the impaired EC maturation, reduced expression of promulticiliogenic genes, and hyperactivated Notch signaling in the lateral ventricles (LVs). Inhibition of Notch signaling restored the ciliogenesis in $M m p 14^{-1-}$ ECs. Taken together, these results revealed a critical role for MT1-MMP in EC ciliogenesis and maturation through suppression of Notch signaling, suggesting that MT1-MMP is essential for postnatal brain development and brain homeostasis.

\section{Results}

Mmp $14^{-1-}$ mice developed communicating hydrocephalus after birth. We previously showed that $M m p 14^{--}$mice exhibit severe growth retardation and die within approximately 2-3 weeks after birth (16). Although obvious morphological abnormalities were hardly observed in the head at birth (Figure 1A), all $\mathrm{Mmp}^{-4^{--}}$mice developed a dome-shaped skull with a compressed volume of cerebellum at later stages, suggesting a progressive increase of intracranial pressure (Figure 1, B and C). Histological analyses revealed a mild dilatation in the LVs and a more prominent dilatation in the third ventricle in $\mathrm{Mmp}^{4^{-/}}$mice (Figure 1C). Ventricular dilatation was initially visible at P3 and became progressively worse from P3 onward, suggesting that loss of MT1-MMP results in a postnatal hydrocephalus.

Aqueductal stenosis is frequently associated with congenital hydrocephalus through the blockage of CSF flow in the narrow channel between the third and fourth ventricles (25). In addition, aqueductal occlusion due to reactive astrogliosis can also give rise to hydrocephalus (26-28). To determine whether the

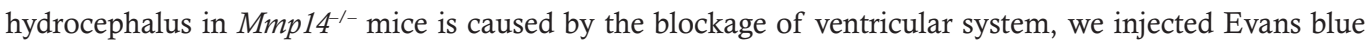
into the left LV to examine whether the dye could reach the fourth ventricle. At P6, the dye was detected at the aqueduct and the fourth ventricles in both WT and mutant mice (Figure 1D). To determine whether there was a subsequent aqueductal obstruction, we examined the flow of CSF through the ventricular system at a later stage (P15) by dye injection and histology analysis. Neither obstruction in the ventricular channel (Figure 1E) nor malformation of the central aqueduct (Figure $1 \mathrm{~F}$ ) was found in $\mathrm{Mmp} 14^{-/}$mouse brain. These data suggest that $M m p 14^{-/-}$mice present communicating hydrocephalus without occlusion.

Mmp $14^{-1-}$ mice exhibited agenesis in corpus callosum and astrocyte hypertrophy. The abnormal development of corpus callosum and astrocytosis have been linked to hydrocephalus $(26,27,29)$. Reactive gliosis has been implicated as a response to the neonatal hydrocephalus. It frequently causes the formation of aqueductal stenosis, which contributes to the development of hydrocephalus (27). To characterize the hydrocephalus in

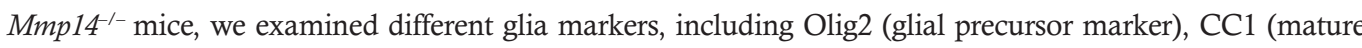
oligodendrocyte cell body marker), myelin basic protein (MBP), and GFAP (astrocytes marker) in serial brain sections from WT and mutant mice (Figure 2). Quantification analyses showed no difference in Olig2 ${ }^{+}$cells in the corpus callosum between WT and $M m p 14^{-1-}$ mice (Figure 2, A, B, and D). However, a significant reduction (42.3\%) in $\mathrm{CC}^{+}$oligodendrocytes in the corpus callosum was observed in MT1-MMP-deficient mice (Figure 2, A and C) compared with their littermate controls. The percentage of $\mathrm{CC}^{+} / \mathrm{Olig} 2^{+}$cells was reduced by $31 \%$ in the corpus callosa of $\mathrm{Mmp} 4^{-/-}$mice (Figure $2 \mathrm{E}$ ). Consistent with this observation, a substantial reduction in MBP immune reactivity in the corpus callosum was found in mutant mice (Figure $2 \mathrm{~F}$ ), suggesting agenesis of the corpus callosum in the absence of MT1-MMP during postnatal development.

In addition, an increased number of $\mathrm{GFAP}^{+}$astrocytes with a hypertrophic morphology at later postnatal stages (P10) (Figure 2, G-I, and Supplemental Figure 1D; supplemental material available online with this article; https://doi.org/10.1172/jci.insight.132782DS1), as well as a transient activation of microglia at P4, was observed in the forebrains of $\mathrm{Mmp} 4^{-/-}$mice (Supplemental Figure $1, \mathrm{~A}-\mathrm{C}$ ), indicating a reactive gliosis in response to hydrocephalus, though it was not sufficient to cause the blockage of CSF flow in mutant brains.

Expression of MT1-MMP in the ventricular system. To understand how loss of MT1-MMP causes hydrocephalus, we examined the pattern of MT1-MMP expression using MT1-MMP/lacZ-knockin $\left(\mathrm{Mmp}_{14}{ }^{+}\right.$ ${ }^{\text {lacZ }}$ ) mice that are indistinguishable from their WT littermates. As shown in Figure 3A, LacZ staining was 
A

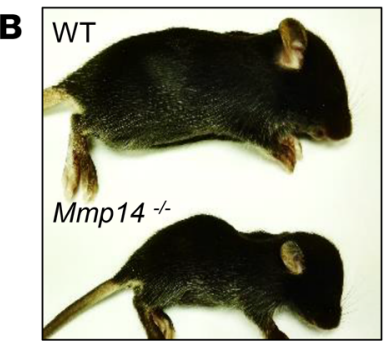

C

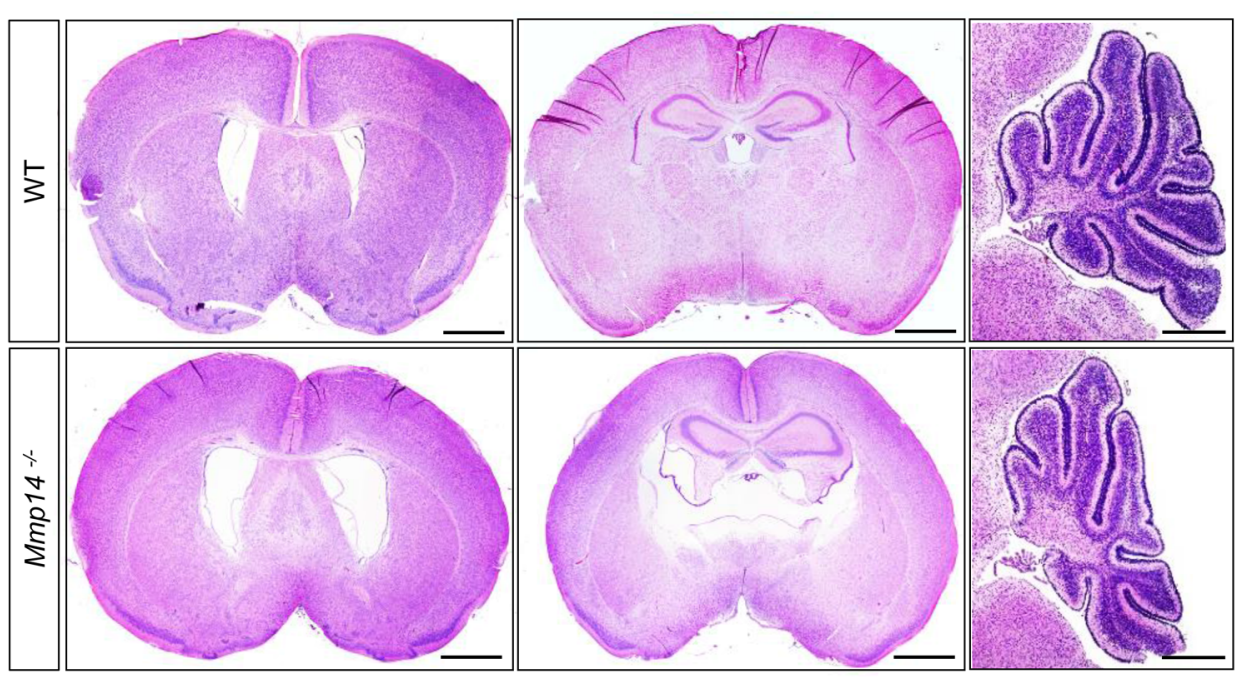

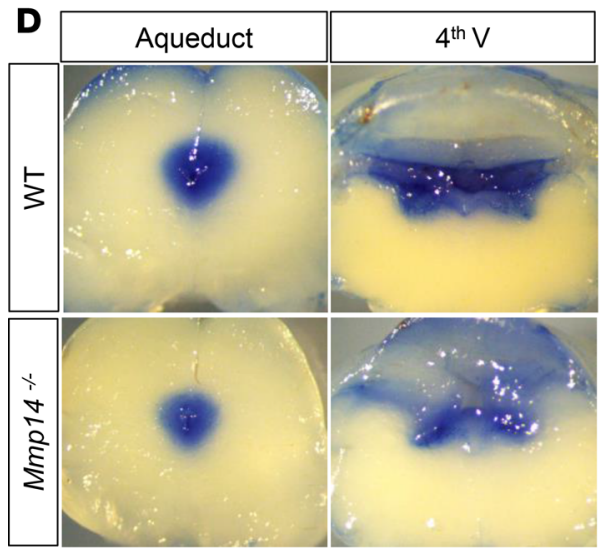
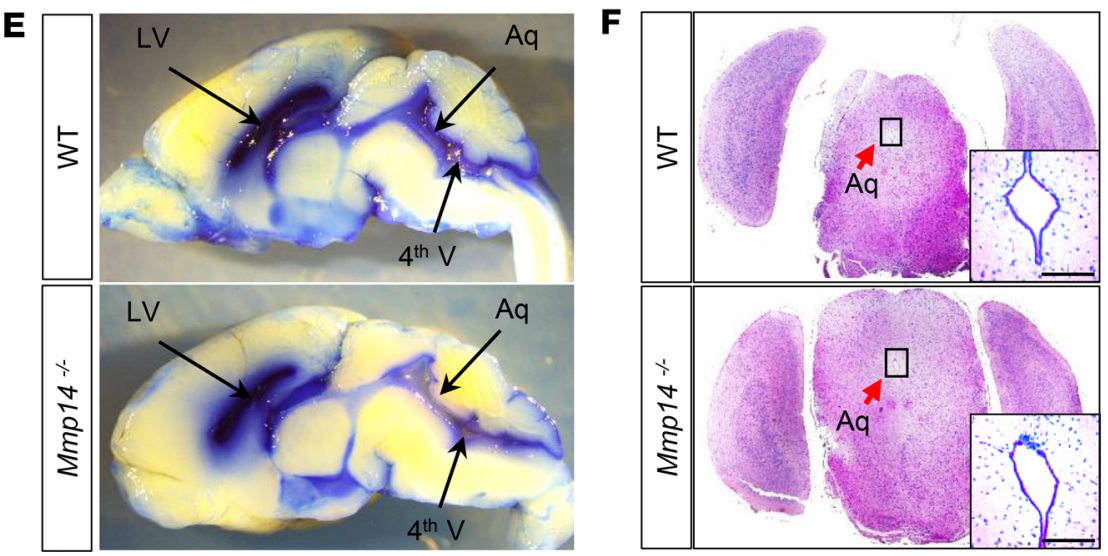

Figure 1. MT1-MMP-deficient mice develop hydrocephalus. (A) A Mmp14 ${ }^{-/-}$mouse at P0 was indistinguishable from a control littermate. (B) A Mmp14 ${ }^{-/-}$ mouse at P15 exhibits a dome-shaped skull, typically observed in progressive hydrocephalus. (C) Coronal sections of P15 mouse brain to examine the LV, the third ventricle, and cerebellum. Scale bar: $500 \mu \mathrm{m}$. (D) Examination of Evans blue dye in the aqueduct and fourth ventricles 5 minutes after injection through an anterior horn of the LV in WT and Mmp14 ${ }^{-/-}$brains at P6. (E) Sagittal slices of brains to examine the distribution of Evans blue dye in the ventricular system in WT and Mmp14 /- brains at P15. (F) H\&E staining of coronal sections of the aqueduct in mouse brain at P15. LV, lateral ventricle; Aq, aqueduct; 4th $\mathrm{V}$, fourth ventricle. Scale bar: $100 \mu \mathrm{m}$.

observed in the ventricular zone (VZ), rostral migratory stream, olfactory bulb, and midbrain in the developing brain but not in the choroid plexus where the CSF is produced. Strong staining of LacZ was observed at the walls of LVs at multiple stages (P3, P6, P10) (Figure 3, B and C), suggesting a potential function of MT1-MMP in LVs. We therefore further investigated the LacZ-expressing cell types in the VZ and subventricular zone (SVZ) in more detail (Figure 3D).

Radial glial cells (RGCs) are the only cell type in the VZ at birth (P0) (30) and the transition from RGCs to ECs occurs between P2 and P6 (31). Loss of the RGCs markers corresponds to the maturation of ECs, which occurs at P7 in mice (31). Thus, we examined the LacZ expression together with RGC and EC markers by double-labeled immunofluorescence in coronal sections of the LV walls. We observed that all $\mathrm{LacZ}^{+}$cells also expressed Sox2, the neural progenitor marker at the VZ regions of postnatal mouse brain (32). At P0, LacZ ${ }^{+}$cells in the VZ were colabeled with the RGC marker brain lipid-binding protein (BLBP). Consistent with a previous report, the EC markers (S100 and vimentin) were not observed at P0 (31). At P10, BLBP expression was reduced substantially, whereas LacZ ${ }^{+}$cells were still observed in the majority of cells in lateral ventricular wall and were positive for $\mathrm{S} 100$ and vimentin. In addition, $\mathrm{LacZ}^{+}$cells were also observed in the SVZ (Figure 3, C and D). Taken together, these data indicated that MT1-MMP is predominantly expressed in the neural progenitors in developing mouse brain and highly expressed in the radial glia and EC lineage lining the LV during postnatal development. 

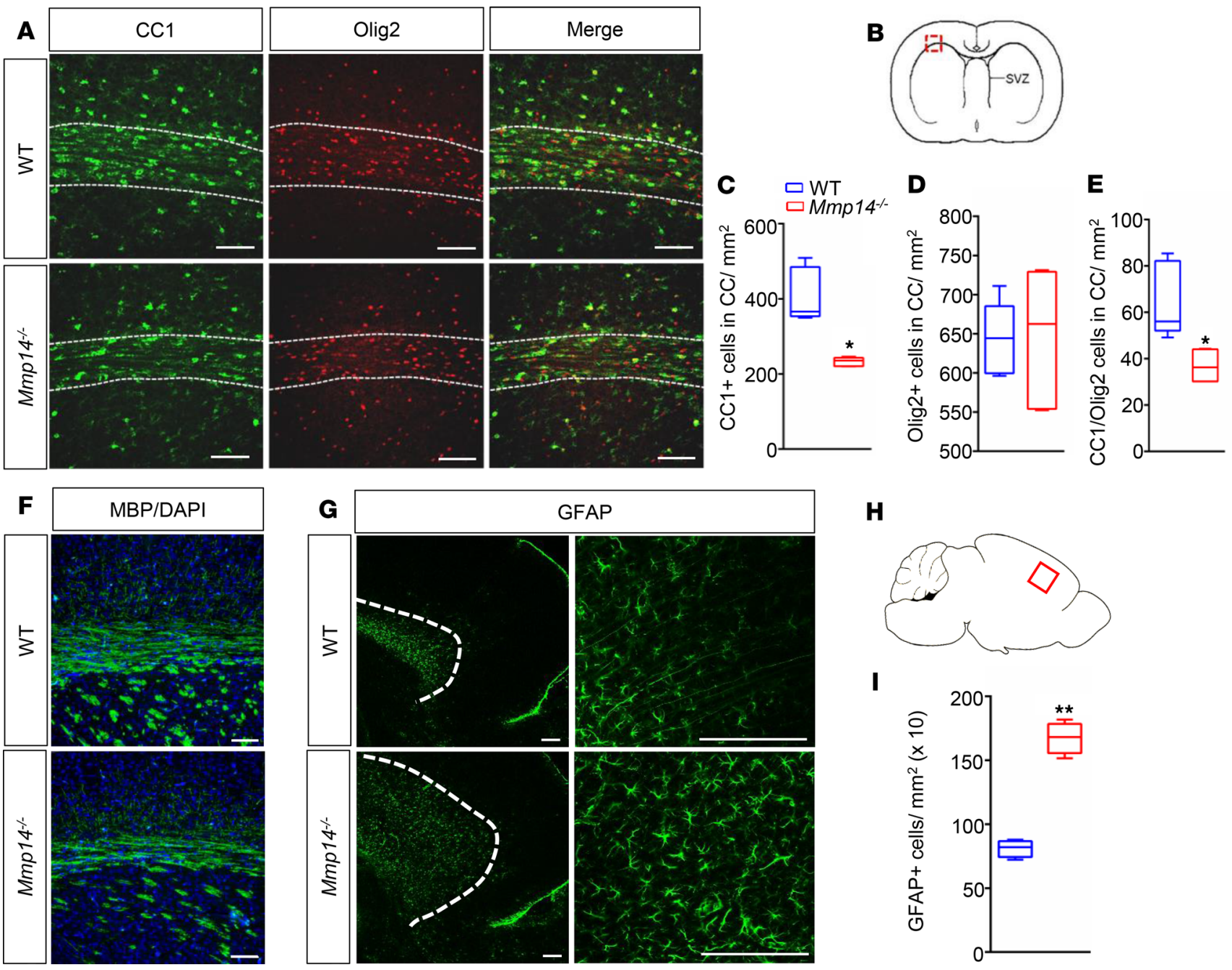

H

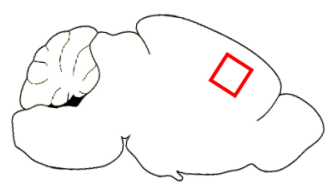

I

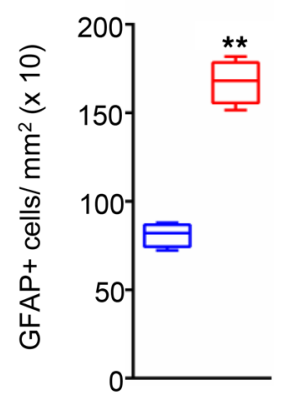

Figure 2. MT1-MMP-deficient mice display corpus callosum agenesis and astrocytosis. (A) Confocal images of WT and Mmp14/- brains in coronal sections stained with antibodies against CC1 (green) and Olig2 (red). (B) The diagram depicts region in the corpus callosum (CC) where high-magnification images were taken. (C and D) Quantification of CC1 ${ }^{+}$cells (C) and Olig2+ cells (D) in CC from WT and Mmp14 ${ }^{-/}$brain sections. Two-tailed Student's $t$ test, ${ }^{*} P<$ 0.05. (E) Quantification of the percentage of $C C 1^{+} / O$ lig2 $2^{+}$cells in CC. Two-tailed Student's $t$ test, ${ }^{*} P<0.05, n=3$. (F) Immunofluorescence staining of myelin basic protein (MBP) of the coronal sections in corpus callosum. (G) Detection of astrocytes by immunofluorescence staining of GFAP in the forebrain at P10. (H) The diagram shows the region in the forebrain where high-magnification images were taken. (I) Quantification of GFAP+ ${ }^{+}$cells in $\mathbf{G}$ by 2-tailed Student's $t$ test, ${ }^{* *} P<0.01, n=4$. Data represent mean \pm SEM. Scale bar: $100 \mu \mathrm{m}$.

Loss of MT1-MMP impairs ependymal ciliogenesis and ciliary function. As MT1-MMP is highly expressed in the ependymal layer (Figure 3) and impaired cilia function in ECs has been shown to be a prevalent cause of early postnatal hydrocephalus $(33,34)$, we wondered if hydrocephalus in MT1-MMP-deficient mice is attributable to the defects in cilia on ECs. To test this, we first examined the P15 brains by immunofluorescence staining of acetylated $\alpha$-tubulin, a motile cilia marker, to depict the cilium bundles when the cilia are fully developed and matured. In WT mice, acetylated $\alpha$-tubulin distributed evenly on ECs lining the LV wall (Figure 4A, arrows), whereas $M m p 14^{-1-}$ ECs exhibited flattened and discontinued staining of acetylated $\alpha$-tubulin (Figure 4A, arrowheads). We subsequently examined the primary and motile cilia on the lateral ventricular surface at multiple stages by scanning electron microscopy. No obvious defect in primary cilia at P0 was observed in the absence of MT1-MMP (Figure 4B). However, the ciliary defects were clearly observed in MT1-MMP-deficient mice by P7. Mutant ECs exhibited considerably shorter cilia and disrupted cilia orientation (Figure 4B, arrowheads), whereas WT ECs displayed evenly distributed cilium bundles with a uniform direction of cilia (Figure 4B, arrows). Quantification revealed that ECs from mutant mice exhibited a significantly reduced number of cilia tufts at the apical surface of LVs $\left(96.9 \pm 4.1 / 0.01 \mathrm{~mm}^{2}\right.$ in WT vs. 
A

Mouse brain saggital section

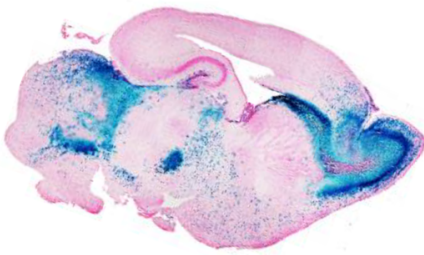

B

Mouse brain coronal section

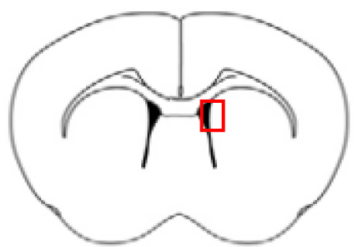

C

P6

D
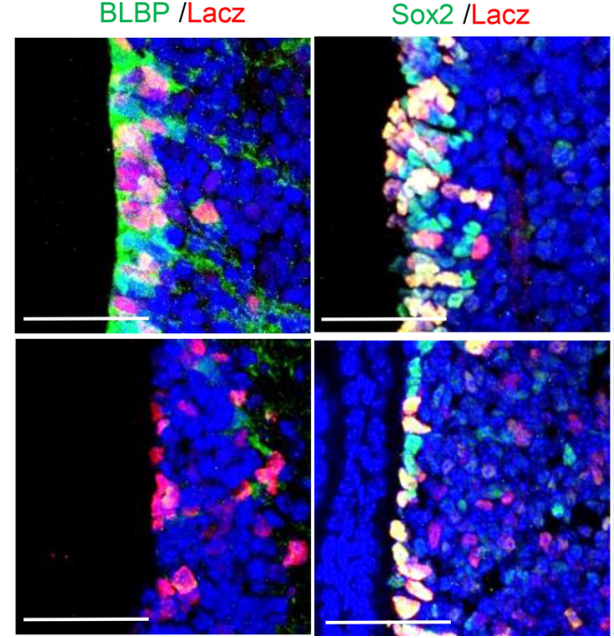

P3

P10
Anterior V-SVZ

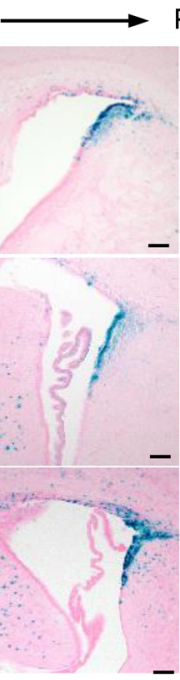

Posterior V- SVZ

Vimentin /Lacz
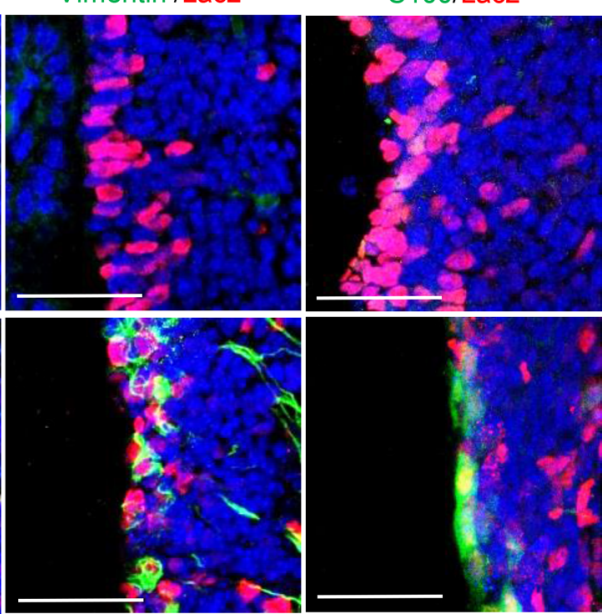

Figure 3. MT1-MMP expression in the wall of the lateral ventricle in Mmp14+/Lacz-transgenic mice. (A) Representative $\beta$-galactosidase staining in the developing brain at P3. (B) Diagram of a coronal section in the dorsal region of a mouse brain showing the LV. High-magnification images of coronal sections with different staining are shown in $\mathbf{C}$ and $\mathbf{D}$. (C) $\beta$-Calactosidase staining (blue) in coronal sections of $\mathrm{Mmp14}^{+/ \text {Lac }}$ mouse brains at P3, P6, and P1O. (D) Representative staining of LacZ, BLBP, Sox2, vimentin, and S100 in coronal sections of LVs from P0 and P10 mouse brains. Scale bar: $50 \mu \mathrm{m}$.

$61.25 \pm 2.2 / 0.01 \mathrm{~mm}^{2}$ in mutant) (Figure 4C) and an increased percentage of cilia tufts with disrupted orientation $(7.9 \% \pm 2.1 \%$ in WT vs. $47.3 \% \pm 12.5 \%$ in mutant) (Figure 4 , B and D) in comparison with those of WT mice. Ciliary defects in the mutant mice progressed until they died at P15. The residual ependymal motile cilia in MT1-MMP-deficient mice, however, retained a normal "9+2" architecture (35), as examined by transmission electron microscopy (TEM) (Supplemental Figure 2). As the disturbed ependymal integrity may impair the circulation of CSF and leads to hydrocephalus $(26,36)$, we then performed $\beta$-catenin and TUNEL staining to assess the ependymal layer integrity (37). Neither gross alterations in $\beta$-catenin pattern nor significant change in apoptosis was observed in the VZ/SVZ (Supplemental Figure 3), indicating a proper organization of the ependymal layer in the absence of MT1-MMP. These data raised the possibility that hydrocephalus in $\mathrm{Mmp14}^{-/}$brains may be attributable to the decreased and disorganized motile cilia in ECs.

To determine whether ciliary defects contribute to abnormal CSF flow in MT1-MMP-deficient mice, we analyzed the ciliary motility ex vivo by tracking microbead movement at the surface of brain ventricles with high-speed video microscopy. As shown in Figure 4E and Supplemental Video 1, the WT ependymal cilia generated a stronger forward force, which propelled a faster movement of the beads, whereas the microbeads showed much slower movements, with a $70 \%$ reduction in mean velocity in mutant samples $(194.3 \pm 6.977 \mu \mathrm{m} / \mathrm{s}$ in WT vs. $55.90 \pm 2.903 \mu \mathrm{m} / \mathrm{s}$ in mutant; Figure 4, E and F, and Supplemental Video 
A

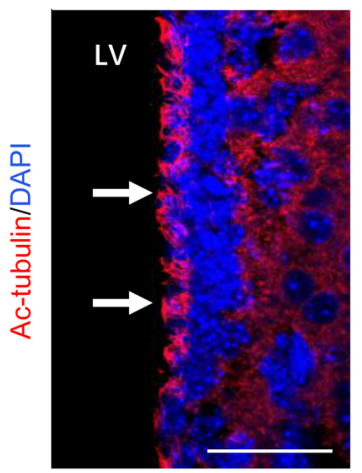

C

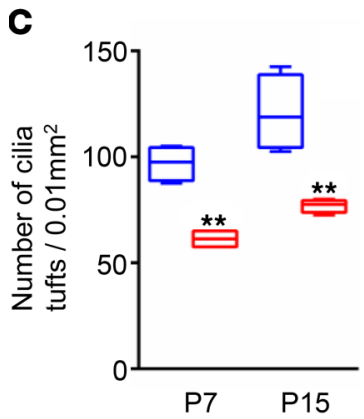

E

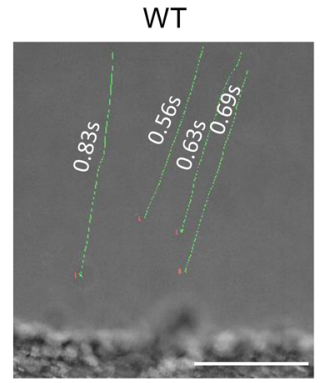

Mmp14

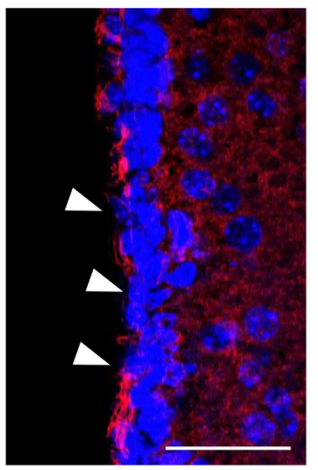

D ${ }^{100}$

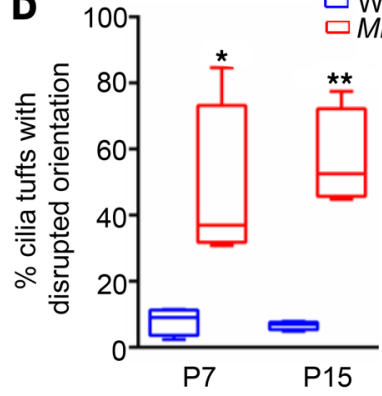

B

WT

§

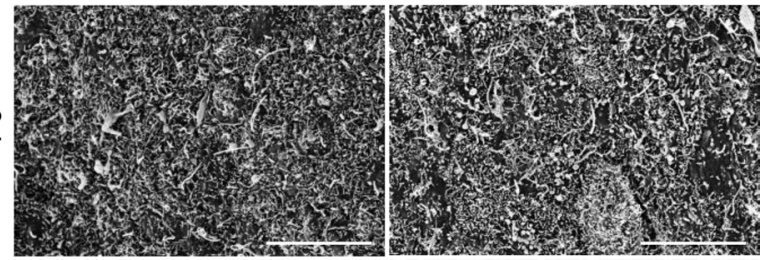

$\check{\Sigma}$
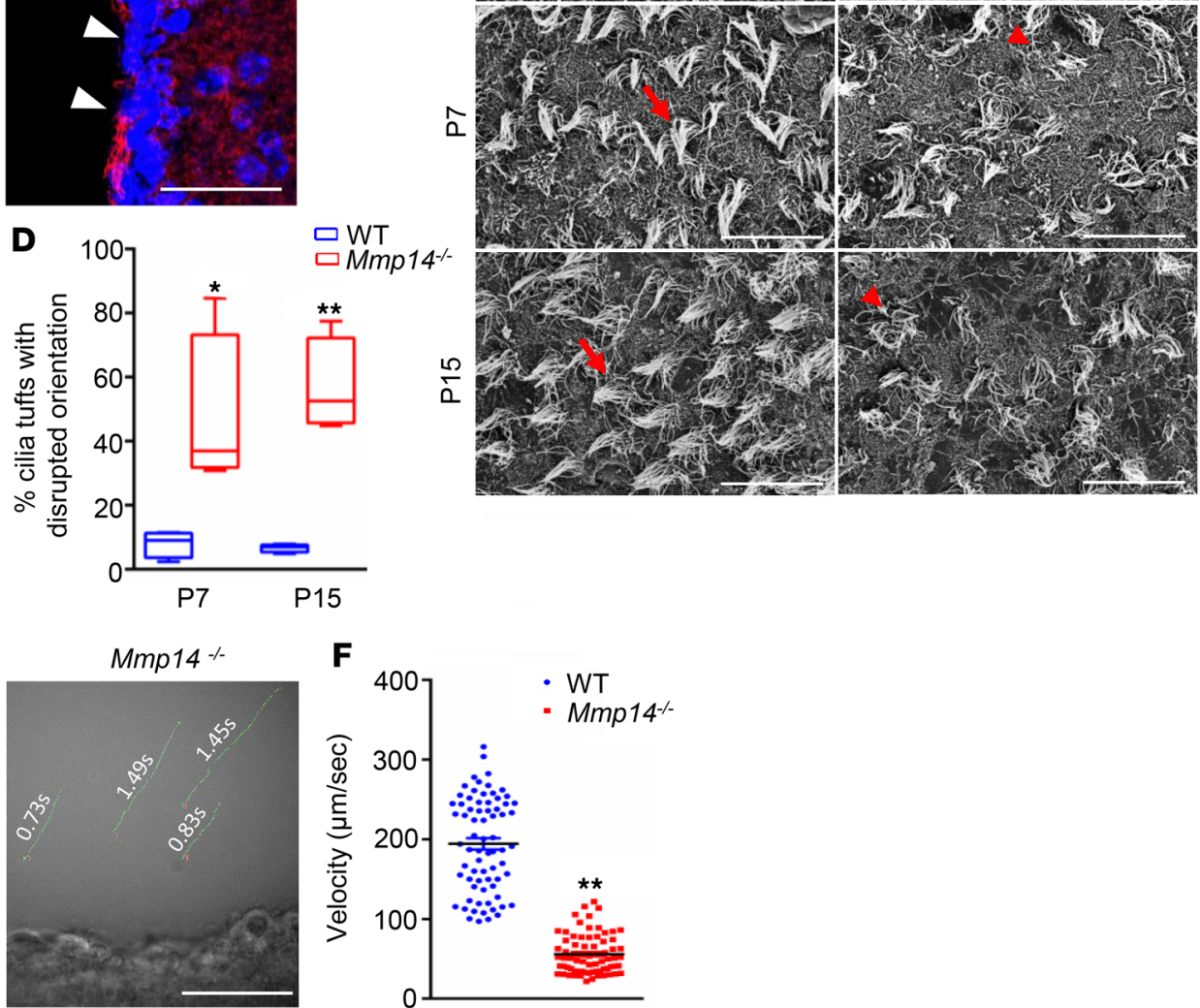

Figure 4. Examination of ependymal cilia in WT and mutant LV. (A) Immunofluorescence staining of acetylated $\alpha$-tubulin in LVs of P15 brains shows evenly distributed cilium bundles in a WT animal (arrows) and flattened and discontinued cilium bundles in a Mmp14 ${ }^{-/}$mouse (arrowheads). Scale bar: $50 \mu \mathrm{m}$. (B) Scanning electron microscopy on the surface of LV walls from WT and Mmp14 ${ }^{-/-}$mice at different postnatal stages. Note the evenly distributed cilium bundles with a uniformed direction of cilia tufts across the LV wall in a WT mouse (arrows) and the sparsely distributed cilium bundles with shorter and fewer cilia in disrupted orientation in a MT1-MMP-deficient mouse (arrowheads). Scale bar: $20 \mu \mathrm{m}$. (C) Quantification of the number of cilia tufts at the surface of the LV in B. Two-tailed Student's $t$ test, ${ }^{* *} P<0.01, n=4$. (D) Quantification of the percentage of cilia tufts with disrupted orientation at the surface of the LV wall in B. Twotailed Student's $t$ test, ${ }^{*} P<0.05$, ${ }^{* *} P<0.01, n=4$. (E) Images overlaid with the movement of the microbeads, as recorded by motion tracking (green lines). Scale bar: $50 \mu \mathrm{m}$. (F) Quantitative analyses of the mean velocity of the microbeads propelled by cilia at the surface of LV walls from WT and mutant brains. A total of 72 microbeads per genotype from 3 independent experiments were analyzed. Two-tailed Student's $t$ test, ${ }^{* *} P<0.01$. Data represent mean \pm SEM.

2). These results demonstrated that loss of MT1-MMP impaired the ependymal ciliogenesis and disturbed the normal CSF flow without affecting the ciliary structure and ependymal integrity.

Loss of MT1-MMP affects ciliary organization and basal body polarity in ECs. The coordinated ciliary beating is crucial for the directional CSF circulation. With the maturation of ECs, the area of apical surface increases and acquires compacted cilia clusters that are initially scattered widely. The motile cilia are then clustered into an off-centered patch at the apical surface of ECs to facilitate coordinated ciliary beating ( 38 , 39). A correlation between defective ciliogenesis and disruption of planar polarity in ependymal motile cilia has been previously described (40). As loss of MT1-MMP results in reduced and disorganized cilia tufts in ECs, we asked whether ciliary defects are associated with disrupted polarity of ECs. The polarity of ECs is normally established at P9 $(41,42)$. We therefore analyzed the morphology and polarity of ciliary basal bodies at $\mathrm{P} 10$ by whole-mount staining of LVs with $\gamma$-tubulin/ $\beta$-catenin antibodies (43). In WT mice, ECs exhibited tightly packed and round basal body clusters of cilia, whereas ECs in MT1-MMP-deficient mice manifested irregular and abnormally elongated patches of cilia (Figure 5A), consistent with the scan- 
ning electron microscopy results. A higher length-to-width ratio of the patches was found in the MT1MMP-deficient ECs (Figure 5B). In addition, MT1-MMP-deficient ECs exhibited a significantly reduced percentage of apical surface coverage by basal body (BB) patches (Figure $5 \mathrm{C}, 34.9 \% \pm 0.38 \%$ of total area in mutant vs. $42.7 \% \pm 0.41 \%$ in WT). The BB patch displacement in MT1-MMP-deficient ECs was significantly increased (Figure 5, D and E, $0.62 \pm 0.005$ in mutants vs. $0.48 \pm 0.006$ in WT). In addition, BB patch angle showed a slight but significantly diverse distribution in MT1-MMP-deficient ECs (Figure 5F). These data clearly showed a defect in planar polarization of BB patches in the absence of MT1-MMP.

Taken together, these data suggest that loss of MT1-MMP affects the intrinsic organization of ciliary basal body patches in ECs and disrupts ciliary patch planar polarity, which in turn disturbs the directional CSF flow in $M m p 14^{-1-}$ mice.

MT1-MMP is essential for EC maturation. ECs are derived from radial glia cells at birth. Defects in either differentiation or maturation of ECs can give rise to hydrocephalus $(4,5,44-46)$. To investigate the potential defects in the differentiation and maturation of ECs, we first examined the density of RGCs at the apical surface of LVs at $\mathrm{P} 0$ by immunofluorescence staining using antibodies against the RGC marker, BLBP. As shown in Figure 6, A and B, WT and mutant mice exhibited similar BLBP ${ }^{+}$cell densities $(203.6 \pm 5.2$ cells $/ \mathrm{mm}$ vs. $187.1 \pm 2.3$ cells $/ \mathrm{mm})$. Vimentin and S100 label the ECs and mature ECs $(44,45,47)$. No significant difference in the number of vimentin ${ }^{+}$ECs was observed between WT and mutant brains at the LV walls $\left(36.4 \pm 5.1 \times 10^{2} \mathrm{~mm}^{2}\right.$ vs. $36.0 \pm 4.4$ cells $\left.\times 10^{2} \mathrm{~mm}^{2}\right)$. However, a significant reduction in the number of $\mathrm{S}_{100}{ }^{+}$cells $\left(23.4 \pm 1.9 \times 10^{2} \mathrm{~mm}^{2}\right.$ in WT vs. $15.4 \pm 1.1$ cells $\times 10^{2} \mathrm{~mm}^{2}$ in mutant $)$ was observed in MT1-

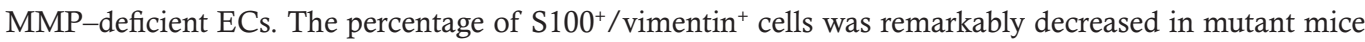
at $\mathrm{P} 10(65.1 \% \pm 4.6 \%$ in WT vs. $41.0 \% \pm 3.5 \%$ in mutant) (Figure $6, \mathrm{C}-\mathrm{F})$, suggesting that the maturation of ECs is MT1-MMP dependent and the hydrocephalic phenotypes in MT1-MMP-deficient mice may be attributable to the compromised maturation of ECs cells in the absence of MT1-MMP.

To investigate how loss of MT1-MMP affects ciliogenesis and the ependymal maturation program, we further assessed the expression of FoxJ1, a transcription factor essential for EC maturation and motile cilia genesis in multiciliated cells, along with its upstream regulator Mcidas (MCI) and downstream targets Centrin2 (Cetn2) and WD repeat domain 78 (Wdr78) $(48,49)$. In line with the defective cilia, we observed that the mRNA of MCI, FoxJ1, Cetn2, and Wrd78 was significantly decreased in the LV walls in the mutant mice at P10 (0.52 \pm 0.06-fold, 0.59 \pm 0.03 -fold, $0.63 \pm 0.06$-fold, $0.70 \pm 0.04$-fold of control) (Figure 6G). A transient reduction in $\mathrm{Myb}$, the gene responsible for centriole amplification at the early postnatal stage, was observed in the LV walls from mutant mice at P3 (0.45 \pm 0.08-fold of control) (Supplemental Figure 4). These results further support the notion that MT1-MMP facilitates maturation of ECs and ciliogenesis at the LV walls in the mouse brain.

MT1-MMP facilitates ciliogenesis of ECs by suppressing Notch signaling. It has been shown that Notch activity suppresses the acquisition of a multiciliated phenotype $(50,51)$. The inhibition of MCI by Notch signaling promotes centriole assembly and motile cilia extension in ECs $(52,53)$. Our previous work showed that MT1-MMP cleaves D111 and negatively regulates Notch signaling to maintain normal B cell development (17). We therefore asked whether MT1-MMP regulates ciliogenesis and EC development in the brain by affecting Notch signaling. As expected, an increased expression of NICD and DLL1 was observed on the mutant ventricular wall (Figure 7A). Consistent with the increased NICD level, elevated expression of Hes5 and Hey1 (1.41 \pm 0.01-fold and $1.47 \pm 0.14$-fold of control at P7; $1.39 \pm 0.05$-fold and $1.92 \pm 0.17$ fold of control at P10) (Figure 7B), the downstream targets of Notch signaling, was observed in the mutant LV, suggesting that loss of MT1-MMP results in a hyperactivation of Notch signaling in the LV walls. To further determine whether defective ciliogenesis in MT1-MMP-deficient ECs is attributable to the excessive Notch signal, we tested if inhibiting Notch signaling will restore the ciliogenesis in MT1-MMP-deficient ECs. ECs isolated from the LVs from both WT and $M m p 14^{-1-}$ brains were taken into culture in the presence and absence of the $\gamma$-secretase inhibitor DAPT. Ciliogenesis was evaluated by the staining of $\gamma$-tubulin, which labels ciliary basal bodies, and acetylated $\alpha$-tubulin, which represents ciliary axoneme when motile cilia are fully developed and matured. Similar to the in vivo observations, defective ciliogenesis was also found in MT1-MMP-deficient ECs. As shown in Figure 7, MT1-MMP deficiency resulted in a significant increase in the number of single ciliated ECs (labeled by $\gamma$-tubulin) $(8.8 \% \pm 0.9 \%$ in WT vs. $22.1 \% \pm 2.0 \%$ in mutant) (Figure $7, \mathrm{C}$ and $\mathrm{D})$ and fewer multiciliated ECs $(57.8 \% \pm 4.4 \%$ in WT vs. $25.2 \%$ $\pm 2.2 \%$ in mutant) (Figure 7, C and E). Mmp $14^{-1-}$ ECs exhibited a remarkable increase in the percentage of multiciliated cells with DAPT treatment $(25.2 \% \pm 2.2 \%$ in mutant ECs treated with DMSO vs. $49.4 \%$ $\pm 4.4 \%$ in mutant ECs treated with DAPT $)$ and a decreased the percentage of monociliated cells $(22.1 \% \pm$ 
A

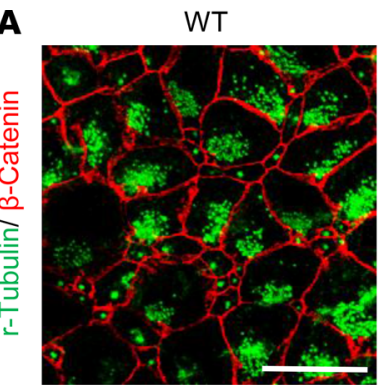

C

c

$\mathbf{E}$

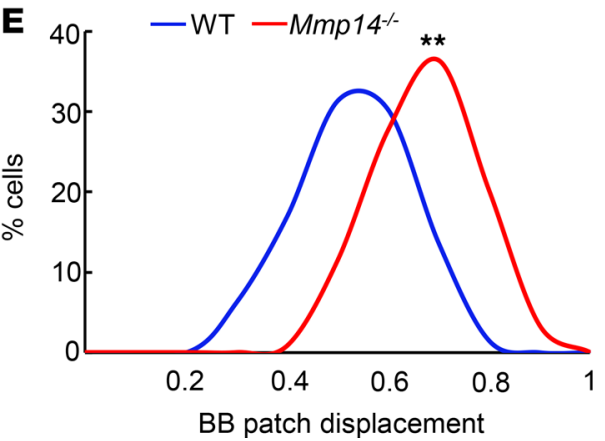

Mmp14

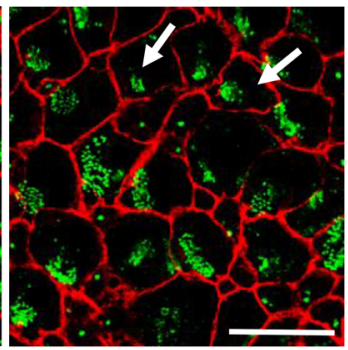

D
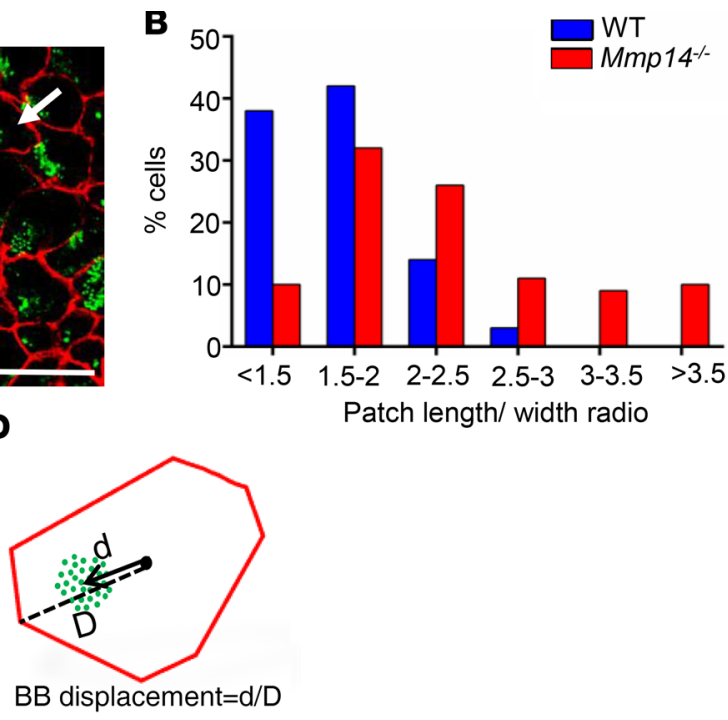

$\mathbf{F}$

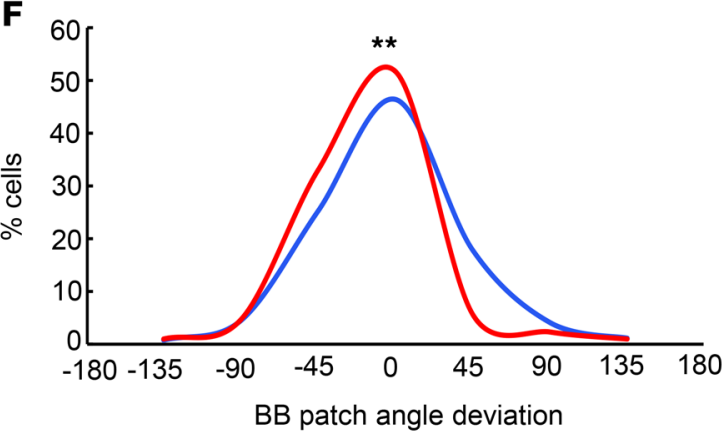

Figure 5. Polarity analyses of basal body patches in ependymal cells. (A) Representative confocal images of wholemount staining of $\gamma$-tubulin (green) and $\beta$-catenin (red) in the walls of LVs from WT and Mmp14 /- brains at P10. Scale bar: $20 \mu \mathrm{m}$. (B) Distribution of the percentage of cells with different length/width ratios of the basal body patches marked by $\gamma$-tubulin staining in A. Contingency table test, $P<0.0001, n=303$ (WT) or $n=339$ (KO) cells from 3 mice per genotype. (C) Quantification of average basal body (BB) patch area in the walls of LVs from WT and Mmp14 ${ }^{-/-}$brains at P10. Unpaired Student's $t$ test, ${ }^{* *} P<0.0001$. (D) Diagram showing the calculation of basal body displacement. The distance between the centroid of the cell and the centroid of the basal body cluster (d, black line) was normalized to the distance ( $D$, broken line) of the line extended to the cell membrane. (E) Histogram of basal body patch displacement in WT (blue) and Mmp14 ${ }^{-/-}$(red) ependymal cells. Unpaired Student's $t$ test, ${ }^{* *} P<0.0001$. (F) Histogram of the distribution of BB patch angles in ependymal cells between WT (blue) and $\mathrm{Mmp14}^{-/-}$(red). Watson's 2-sample $\mathrm{U}^{2}$ test, ${ }^{* *} P<0.01, n=269$ (WT) or $n=300$ (KO) cells from 3 mice per genotype.

$2.0 \%$ in mutant ECs treated with DMSO vs. $9.3 \% \pm 1.4 \%$ in mutant ECs treated with DAPT) (Figure $7, \mathrm{C}$ and D). In addition, while DAPT treatment did not significantly increase the cilia length in WT ECs (7.20 $\mu \mathrm{m} \pm 0.07 \mu \mathrm{m}$ in DMSO treatment vs. $7.39 \mu \mathrm{m} \pm 0.06 \mu \mathrm{m}$ in DAPT treatment), the cilia length was restored remarkably in mutant ECs treated with DAPT $(3.29 \mu \mathrm{m} \pm 0.07 \mu \mathrm{m}$ in DMSO treatment vs. $6.60 \mu \mathrm{m} \pm 0.07$ $\mu \mathrm{m}$ in DAPT treatment) (Figure 7, C and F). These results demonstrated that hyperactivated Notch signaling in the absence of MT1-MMP is responsible for the defective ciliogenesis in ECs.

\section{Discussion}

Hydrocephalus, a common defect frequently occurring in infants, is characterized by the increased head size, impaired vision, poor coordination, seizures, poor appetite, and urinary incontinence. Hydrocephalus is usually accompanied by disrupted neural cell determination in the SVZ, reduced subcommissural organ, shrunken corticospinal tract, or deficiency in corpus callosum (54). In mice, the most prominent phenotypes are dome-shaped skull and enlarged ventricles caused by the accumulation of CSF, due to either the 

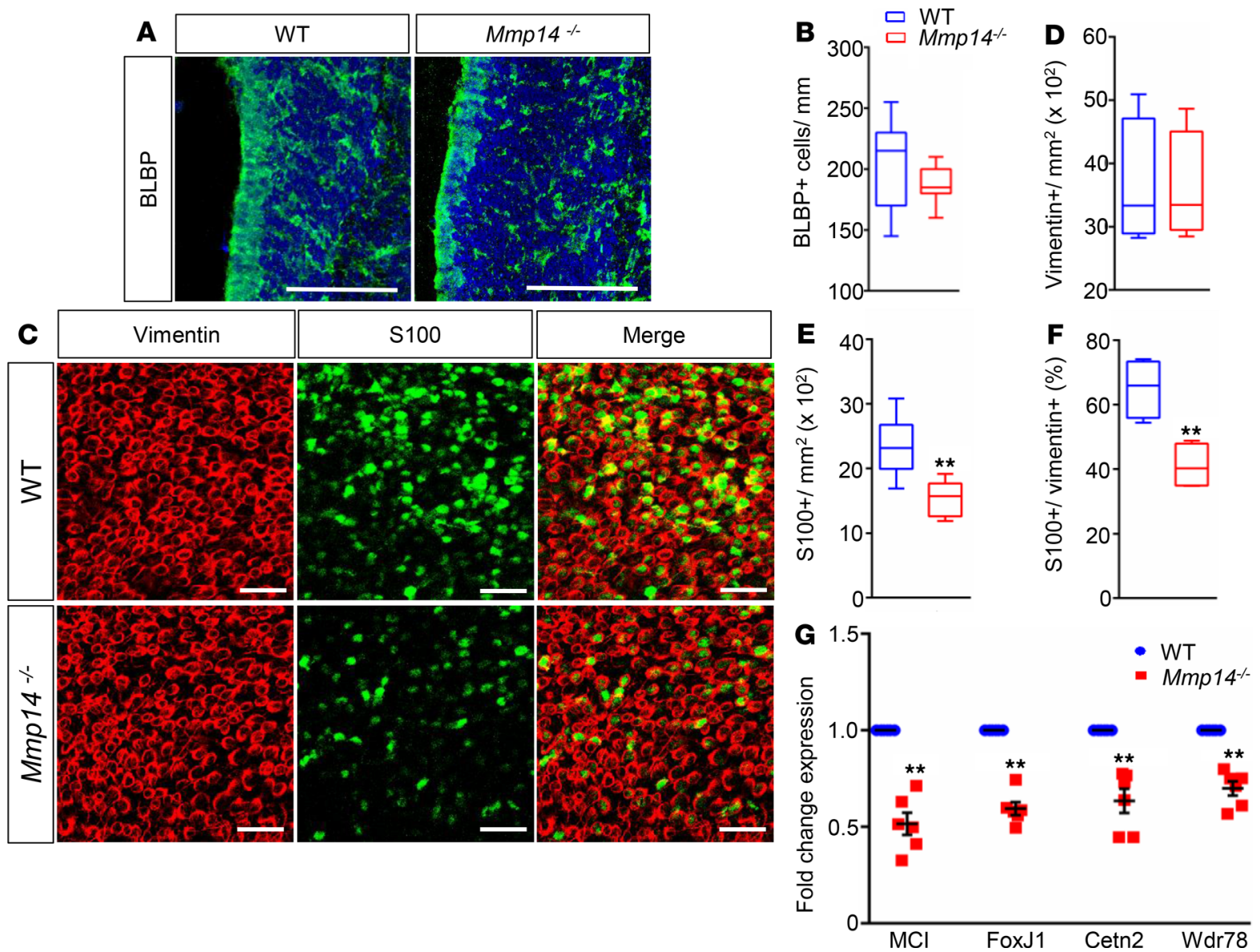

Figure 6. Examination of ependymal cell maturation and ciliogenesis in WT and Mmp14-/- brains. (A) Representative confocal images of immunofluorescence staining of BLBP (green, radial glial cell marker) in WT and $\mathrm{Mmp}^{14^{-/-}}$brain sections at PO. Scale bar: $100 \mu \mathrm{m}$. (B) Quantification of BLBP ${ }^{+}$cells lining the LV walls in WT and Mmp14 ${ }^{-/}$mice at PO. Two-tailed Student's $t$ test, $P>0.05, n=3$. (C) Whole-mount staining of vimentin (red) and S100 (green) in the LV walls from WT and Mmp14 ${ }^{-/-}$mouse brains at P10. Scale bar: $50 \mu \mathrm{m}$. (D) Quantification of vimentin ${ }^{+}$cells in whole-mount staining of the LV wall. Two-tailed Student's $t$ test, $P>0.05, n=6$. (E) Quantification of $5100^{+}$cells in whole-mount staining of the LV wall. Two-tailed Student's $t$ test, ${ }^{* *} P<0.01$, $n=6$. (F) Percentage of $5100^{+} /$vimentin+ cells in the LV wall. Two-tailed Student's $t$ test, ${ }^{*} P<0.01, n=6$. (G) Fold changes in mRNA level of promulticiliogenic genes (MCl, Fox)1, Cetn2, and Wdr78) at the LV walls at P10. Levels of gene expression in WT samples were designated as 1. Two-tailed Student's $t$ test, ${ }^{* *} P<0.01, n=6$. Data represent mean \pm SEM.

obstruction of the ventricular system or defects in the ECs. For example, mutations in genes encoding for structural and functional proteins of cilia, including TMEM67, MKS1, SNX27, and CC2D2A, result in congenital hydrocephalus $(5,55,56)$.

Loss of MT1-MMP results in a dome-shaped skull. In this study, we showed that loss of MT1-MMP gives rise to congenital hydrocephalus. Mmp14 $14^{--}$mice exhibited enlarged ventricular cavities, agenesis of the corpus callosum, and astrocyte hypertrophy. Mutant mice manifested a communicating hydrocephalus without obvious obstruction in the ventricular channels demonstrated by H\&E staining and Evans blue dye injection (Figure 1). We observed that MT1-MMP is predominantly expressed in the radial glia at birth, which is the only cell type in the LV wall at $\mathrm{P0}$, and in the EC lineage lining the LV during postnatal development but not in the choroid plexus where the CSF is produced. We therefore suspected that MT1-MMP affects ECs whose functional defect is closely associated with the pathogenesis of hydrocephalus. Indeed, Mmp $14^{-/-}$ECs exhibited reduced and disorganized motile cilia, though the inner structure was not affected. The ciliary defects generated an impaired CSF flow at the surface of LV (Figure 4 and Supplemental Video 2) that is likely the major contributor to the hydrocephalus in MT1-MMP-deficient mice (57, 58). These data suggest that MT1-MMP is critical for the control of ciliogenesis in ECs.

The planar polarity of ECs controls the coordinated ciliary beating and the directional CSF flow, therefore making the polarity essential for brain function (40). Several studies have shown that planar cell polarity (PCP) genes (Celsr1, Fzd3, Vang12, and Dvl) regulate cilia positioning in the ependymal apical 
A

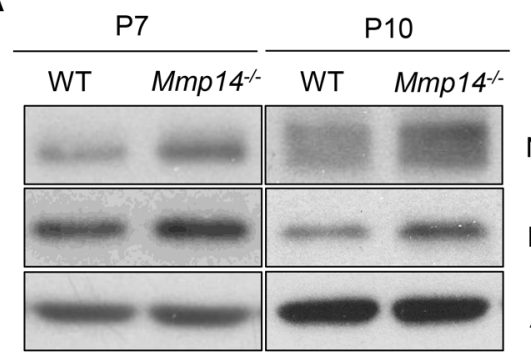

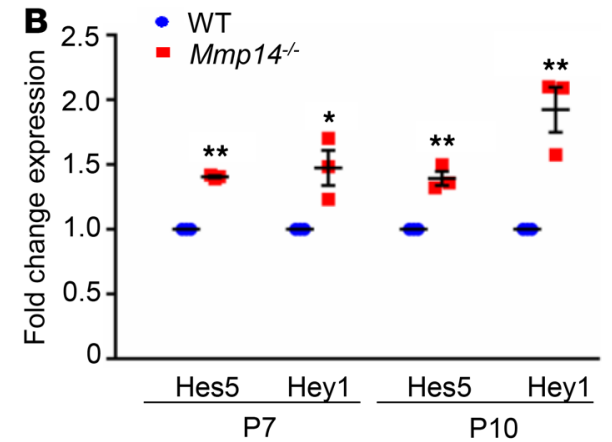

C
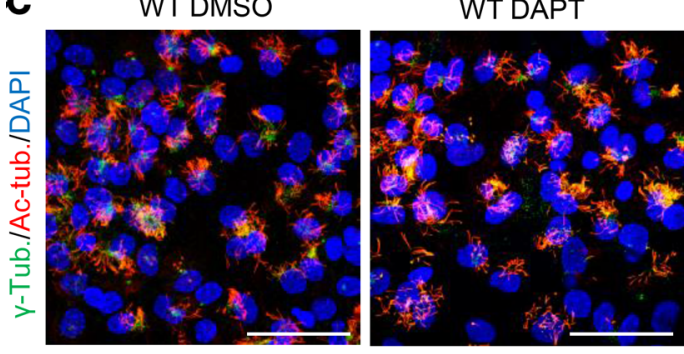

E

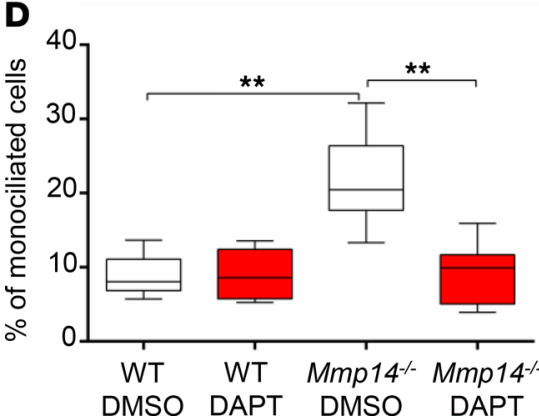

NICD

DLL1

Actin
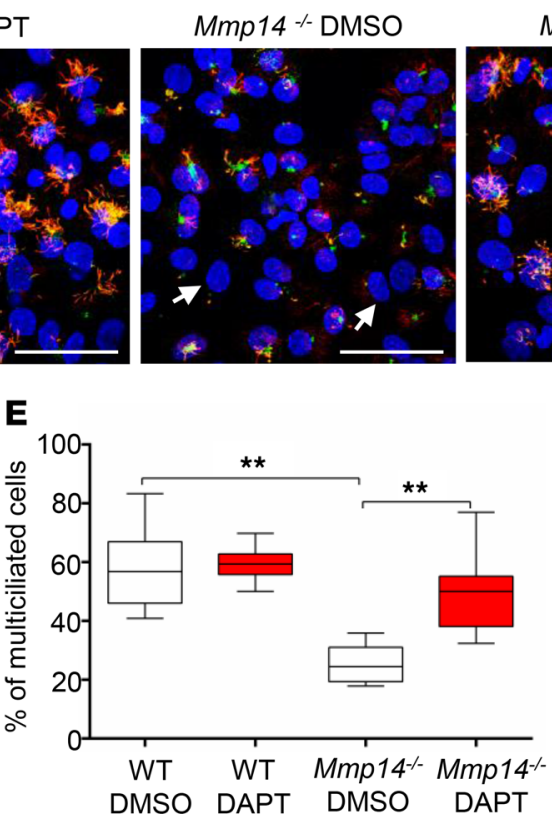

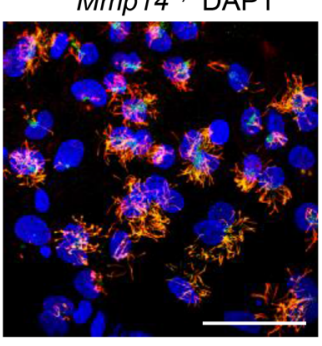

F

Figure 7. Examination of Notch signaling in lateral ventricles and inhibition of Notch signaling in cultured ECs isolated from mouse brains. (A) Representative Western blotting shows the NICD, DLL1, and $\beta$-actin expression of LVs from WT and Mmp14 ${ }^{-/}$brains at P7 and P10. (B) Fold change in mRNA levels of Hes5 and Hey1 in LVs of WT and Mmp14 ${ }^{-/-}$brains at P7 and P10. Gene expression levels in WT samples were designated as 1. Two-tailed Student's $t$ test, ${ }^{*} P<0.05$, ${ }^{* *} P<0.01, n=3$. (C) Immunofluorescence staining of $\gamma$-tubulin (green) and acetylated $\alpha$-tubulin (red) in WT and Mmp14 ${ }^{-/-}$ECs cultured in the presence or absence of DAPT for 7 days without serum. Arrows indicate ECs with single cilium. Scale bar: $50 \mu \mathrm{m}$. (D and E) Quantification of the percentage of monociliated and multiciliated cells in cultured ECs in the presence or absence of DAPT. (F) Quantification of cilia length in cultured ECs in the presence or absence of DAPT. A total number of 500 cilia per group from 3 independent experiments were measured. One-way ANOVA followed by Tukey's comparison test, ${ }^{* *} P<0.01$. Data represent mean \pm SEM.

domain and deletion of PCP genes leads to severe hydrocephalic phenotypes $(4,38,44)$. To investigate how MT1-MMP affects the development of ECs and whether the ciliary disorganization in Mmp14 ${ }^{-/}$mice is a consequence of disrupted PCP, we characterized the basal bodies of motile cilia. The BB patch displacement was significantly increased in the ECs in $\mathrm{Mmp} 4^{-/}$mice. The BB patch angles, a readout of translational polarity, was disturbed, suggesting that MT1-MMP is indispensable in the planar polarization of BB patches. Previous reports showed that MT1-MMP can cleave PCP protein PTK7 and directly regulate the directional cell migration and tumor invasion $(59,60)$. It is unclear whether a defect in PTK7 cleavage may also contribute to the disrupted cilia orientation in ECs in $\mathrm{Mmp}^{14^{-1-}}$ mice.

On the other hand, many mutant ECs manifested dispersed and abnormally elongated BB patches as well as a lower percentage of apical surface coverage by basal bodies, a phenomenon frequently found to correspond to the immature multiciliated ECs $(39,46)$. Indeed, we observed a decreased expression of $\mathrm{Myb}$, the gene responsible for the centriole amplification during early multiciliogenesis (61), in the LV walls from mutant brains, which may contribute to the abnormal BB patches in mutant mice. To substantiate the findings of impaired differentiation or maturation in MT1-MMP-deficient ECs, we examined the expression of RGC marker BLBP at the LV walls at birth, followed by the examination of vimentin and S100 by whole-mount staining of LV at different postnatal stages. ECs lining the LV during the first week of post- 
natal life are differentiated from the radial glia and acquire their final characteristics by the second week (31). Vimentin immunoreactivity is mainly detected in ECs at the LV walls, whereas S100 is considered as a marker for mature multiciliated ECs $(47,62)$. Interestingly, MT1-MMP deletion did not result in decreased $\mathrm{BLBP}^{+}$cells or vimentin ${ }^{+} \mathrm{EC}$, suggesting that the differentiation of RGCs to EC lineage was not disturbed in MT1-MMP-null mice. These data also indicate that the integrity of the ependymal layer is maintained in LVs of $\mathrm{Mmp}^{-4^{--}}$mice, a finding that is also supported by the normal $\beta$-catenin and TUNEL staining. However, a significant reduction in $\mathrm{S} 100^{+}$cells was found in the LV walls in mutant mice compared with that in WT mice at P10, suggesting that MT1-MMP is required for the maturation of ECs.

FoxJ1 is a key transcriptional factor required for centriole assembly during ciliogenesis; it controls the differentiation and maturation of ECs $(48,49)$. FoxJ1 can be activated directly by MCI, a key regulator for genesis of multiciliated ECs, which is inhibited by Notch signaling $(53,63)$. Consistent with the decreased number of $\mathrm{S} 100^{+}$cells in the LV walls in the mutant brains, we observed a reduced expression of MCI and FoxJ1, along with their downstream targets (Cetn2 and Wdr78), in the mutant LVs, further supporting the notion that MT1-MMP is required for ciliogenesis and ECs development.

Notch signaling is important for both survival and maintenance of stem cell quiescence in the neural lineage. ECs express the Notch1 receptor as well as its preferred ligand delta-like1 (D111) (64). It has been reported in several hydrocephalic models that Notch signaling activation suppresses the acquisition of a multiciliated phenotype and inhibition of excessive Notch signal results in the upregulation of MCI and FoxJ1, promoting ciliogenesis and maturation of ECs (5, 39). Given that MT1-MMP negatively regulates Notch signaling by cleaving D111 to maintain normal B cell development (17), it is plausible to speculate that MT1-MMP likely affects ciliogenesis and EC development through modulating Notch signaling. In line with the downregulated Foxj1 expression in LVs in mutant brains, we observed hyperactivation of Notch signaling represented by increased levels of NICD, D111, and their downstream targets (Hes5 and Hey1), further supporting that MT1-MMP affects the maturation of ECs and ciliogenesis through suppressing Notch signaling by processing D111. Indeed, the specific Notch signaling inhibitor, DAPT ( $\gamma$-secretase inhibitor), drastically rescued the ciliary defects in the mutant ECs in terms of the number of multiciliated cells and ciliary length, demonstrating that MT1-MMP regulates ciliogenesis and maturation of ECs, at least in part, if not entirely, by suppressing Notch hyperactivation. Our data identified a potentially novel function for MT1-MMP in regulating ciliogenesis in ECs during brain development and provided insights into the molecular pathogenesis of congenital hydrocephalus.

\section{Methods}

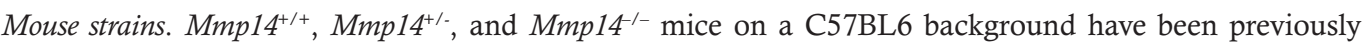
described $(18,21)$. MT1-MMP ${ }^{+/ L a c Z}$ mice were provided by Takeharu Sakamoto from the University of Tokyo (Tokyo, Japan) (65). Both male and female animals were used in experiments. The age at which mice were used for experiments is shown in the figure legends.

Ventricular dye injections. The CSF circulation was assessed as described previously $(6,7)$. Briefly, P6 and P15 Mmp $14^{+/-}$and $\mathrm{Mmp}_{14^{-/}}$mice were deeply anesthetized by intraperitoneal injection of ketamine and xylazine. Evans blue dye ( $5 \mu 1,4 \%$ in PBS) was injected into the LV using a $0.1-\mathrm{ml}$ syringe. Mice were then sacrificed 5 minutes after the injection. Brains were fixed in 4\% PFA overnight and then cut into 2-mm-thick slices.

$\beta$-Galactosidase staining. Dissected brains were postfixed for 1 hour at room temperature and sectioned using a vibratome. The $\mathrm{X}$-gal solution was freshly prepared by dissolving X-gal powder into dimethyformamide and added into the staining solution containing $1 \mathrm{mg} / \mathrm{mL} \mathrm{X-gal,} 5 \mathrm{mM} \mathrm{K}_{3} \mathrm{Fe}[\mathrm{CN}]_{6}, 5 \mathrm{mM} \mathrm{K}_{4} \mathrm{Fe}[\mathrm{CN}]_{6}$, and $2 \mathrm{mM} \mathrm{MgCl}$ in PBS. The samples were incubated for approximately $12-16$ hours in the dark at $37^{\circ} \mathrm{C}$. The tissues were washed and fixed on next day and then dehydrated and embedded for sectioning. The sections were counterstained in Eosin Y (MilliporeSigma, HT110216) for imaging.

Histology and immunofluorescence. Mouse brain samples were fixed in $4 \% \mathrm{PFA}$ overnight at $4^{\circ} \mathrm{C}$, embedded in gelatin or dehydrated, and embedded in wax. Paraffin and cryosections were generated at $8 \mu \mathrm{m}$ and $20 \mu \mathrm{m}$, respectively. Paraffin sections were used in H\&E staining and imaged under a bright-field microscope (BX51, Olympus) equipped with a camera. The sections were immunostained using primary antibodies against the following proteins: $\beta$-catenin (BD Transduction, 610154); acetylated- $\alpha$-tubulin (MilliporeSigma, T7451); LacZ (Abcam, ab134435); Vimentin (Santa Cruz, sc-7557); BLBP (MilliporeSigma, ABN14); Olig2 (MilliporeSigma, AB9610), CC1 (Abcam, Ab16794), MBP (MilliporeSigma, AB5864), Sox2 (Neuromics, GT15098), and GFAP (DAKO, Z0334). Secondary antibodies coupled to Alexa Fluor 
488 or 568 of donkey anti-mouse, rabbit, or goat (Invitrogen) were diluted at 1:500 and incubated at room temperature for 1 hour. Sections were mounted with mounting medium containing DAPI (MilliporeSigma). Confocal images were acquired by a Zeiss LSM780 microscope.

Whole-mount immunostaining. After cervical dislocation, the brain was removed from the skull and whole mounts of the LV walls were freshly dissected as described previously (43). The exposed walls were then fixed overnight at $4^{\circ} \mathrm{C}$ in $4 \%$ paraformaldehyde in $0.1 \%$ Triton X-100/PBS. The whole-mount samples were incubated in primary antibodies in $0.5 \%$ Triton X-100/PBS, including anti- $\gamma$-tubulin (MilliporeSigma, T5192); anti- $\beta$-catenin (BD Transduction, 610154); anti-Vimentin (Santa Cruz, Sc-7557); and anti-S100 (MilliporeSigma, S2644), for 24 hours at $4^{\circ} \mathrm{C}$. Secondary antibodies coupled to Alexa Fluor 488 or 568 of donkey anti-mouse, -rabbit, or -goat (Invitrogen) were diluted at 1:600 and incubated at room temperature for 1.5 hours. Confocal images were acquired by a Zeiss LSM780 microscope.

Electron microscopy. Mouse LV walls were fixed overnight at $4^{\circ} \mathrm{C}$ in $2.5 \%$ glutaraldehyde in $0.1 \mathrm{M}$ phosphate buffer $\mathrm{pH}$ 7.4. For scanning electron microscopy, LV walls were dehydrated through a series of graded ethanol $(30 \%, 50 \%, 75 \%, 95 \%$, and 100\%) and dried with a critical-point dryer (Praeparation, CPD 030). Samples were mounted and coated with platinum with sputter coater, and images were captured with a Leo 1530 FEG scanning electron microscope. Blocks for TEM were rinsed, postfixed in osmium tetroxide, dehydrated by a series of ethanol and propylene oxide, and then embedded in epoxy resin. After polymerization, blocks were sectioned at $100 \mathrm{~nm}$ and stained with uranyl acetate and lead citrate. Finally, sections were dried and observed with a Philips CM100 transmission electron microscope.

TUNEL staining. An In Situ Cell Death Detection Kit (11684795910, Fluorescein, Roche) was used to detect cells under apoptosis. The experiment was performed on paraffin sections according to the manufacturer's instructions. Apoptotic cells were counted blind on 3 comparable sections per mouse brain $(n=4)$.

Video microscopy analysis of ependymal ciliary function. Fresh brains were dissected from 12-day-old animals. One-mm-thick coronal sections from LV walls were obtained and transferred to a 35-mm glass microwell dish in DMEM at $37^{\circ} \mathrm{C}$. Before video microscopy analysis, a small amount polystyrene beads (Invitrogen, $2 \mu \mathrm{m}$ ) was added and mixed with the medium. Bead movement was recorded by a high-speed video camera (Nikon Ti2-E wide-field microscope) with a $\times 100$ oil immersion objective. Images were captured at 30 frames per second, and the mean velocity of each tracked microbead was calculated using MetaMorph software.

EC culture. LVs were dissected from P0 or P1 mice in pro-cold PBS and digested with papain (MilliporeSigma, P4762) with DNAse I (Stemcell Technologies, 07469) in DPBS at $37^{\circ} \mathrm{C}$ for 15 minutes followed by centrifugation. The cells were resuspended in DMEM with 10\% FBS and 1\% Penicillin/Streptomycin (Gibco, 151400) and plated on Poly-L-Ornithine-coated (MilliporeSigma, P4957) coverslips in 24-well plate. The cells isolated from a single brain were seeded into 2 wells of a 24-well plate. After 24 hours to 5 days. when the cells reach $100 \%$ confluence, the medium was changed to DMEM without serum to induce EC differentiation. For the ciliogenesis rescue experiment, DAPT (Adipogen) was used at $20 \mu \mathrm{M}$ in DMSO, and control cells were treated with equivalent volume of vehicle (DMSO). The medium was changed every 3 days.

Quantitative PCR. Total RNA was extracted from mouse LV walls using Trizol reagent (Invitrogen) and reversed transcribed into cDNA using M-MLV reverse transcriptase (Promega). Real-time PCR reactions were performed in StepOnePlus Real-time PCR System (Applied Biosystems) with the SYBR Green PCR master mix (Takara). Triple qRT-PCRs were performed for each primer pair. The primer sequences were as follows: MCI (forward, 5'-GGCCTCAGTGCTGGATAAGC-3', reverse, 5'-TAGGGTCACGATTGTGCAGG-3'); Foxj1 (forward, 5'-CTTCCGCCATGCAGACCCCA-3', reverse, 5'-CGGGCAAAGGCAGGGTGGAT-3'); Cetn2 (forward, 5'-ACCAGAAACAGGAAATCCGGG-3', reverse, 5'-CСТCATTGCCACCTTCAGTTC-3'); Wdr78 (forward, 5'-ACTAGCCGGGGAGACGTAAT-3', reverse, 5'-TGATGTTGGTCCCTTTCCGT-3'); Hes5 (forward, 5'-CAAGGAGAAAAACCGACTGCG-3', reverse, 5'-GCGAAGGCTTTGCTGTGTTT-3'); Hey1 (forward, 5'-TACCCAGTGCCTTTGAGAAG-3', reverse, 5'-AACCCCAAACTCCGATAGTC-3') (39); Myb (forward, 5'-AGCAGGCATTACCAACACAGA-3', reverse, 5'-CTGCTGAGATCACACCACGA-3'); and GAPDH (forward, 5'-GAGCCAAAAGGGTCATC-3', reverse, 5'-GTGGTCATGAGTCCTTC-3'). Gene expression was normalized with GAPDH mRNA levels.

Immunoblot analysis. LVs isolated from WT and $\mathrm{Mmp}^{14^{-1}}$ mouse brains were lysed in lysis buffer contains 50 mM Tris-HCL ( $\mathrm{pH} 7.5$ ), $150 \mathrm{mM} \mathrm{NaCl}, 1 \%$ Nonidet $\mathrm{P} 40,0.5 \%$ sodium deoxycholate, and protease inhibitor cocktail (Roche). Protein lysate was mixed with loading buffer and electrophoresed in SDS-PAGE 
and transferred onto nitrocellulose membranes. Membranes were incubated with primary antibody (anti- $\beta$ actin [MilliporeSigma, A5316], anti-NICD [Cell Signaling, 4147], and anti-D111 [Santa Cruz, H265]) after blocking and then analyzed by immunoblot analysis.

Statistics. All data are expressed as mean \pm SEM. Student's unpaired 2-tailed $t$ test was used for comparisons between 2 groups, and 1-way ANOVA followed by post hoc comparisons with Tukey's correction was used for multiple comparisons. Statistical significance was defined as $P<0.05$. The BB displacement was calculated as the distance between the cell center and the BB patch center divided by the distance from the cell center to the membrane (38). To examine a cell's BB patch angle, a vector was drawn from the cell center to the $\mathrm{BB}$ patch center in images aligned with the anterior side to the left, and the angle was compared between WT and mutant mice using Watson's 2-sample $\mathrm{U}^{2}$ test. The distance and vector angles were measured using ImageJ software (NIH) (66).

Study approval. All animal experiments were approved by the Committee on the Use of Live Animals for Teaching and Research of the University of Hong Kong and carried out according to its guidelines and regulations.

\section{Author contributions}

$\mathrm{ZZ}$ and XL conceived and supervised the project. ZJ designed and performed the experiments. ZZ and ZJ analyzed the data. JZ, XQ, HZ, and GJ initiated the investigation and helped to collect some of the data. $\mathrm{BG}$ advised on PCP analysis. ZZ and ZJ wrote the manuscript.

\section{Acknowledgments}

This work was supported by Hong Kong Research Grant Council (HKU 787011M; T13-607/12-R); University Research Committee on Research and Conference Grants (HKU 201001176085); and National Science Foundation of China (31271492, 81671399, and 8197329). We thank the staff of Faculty Core Facility, University of Hong Kong, Hong Kong, China for their technical support and providing the polystyrene beads; Liliana Osorio and Zonghua Li for fruitful discussion; and Haitao Sun for technical assistance.

Address correspondence to: Zhongjun Zhou, School of Biomedical Sciences, Li Ka Shing Faculty of Medicine, University of Hong Kong, 21 Sassoon Road, Hong Kong, China. Phone: 852.64483946; Email: zhongjun@hku.hk.

1. Ruggeri G, et al. Bi-allelic mutations of CCDC88C are a rare cause of severe congenital hydrocephalus. Am JMed Genet A 2018;176(3):676-681.

2. Symss NP, Oi S. Theories of cerebrospinal fluid dynamics and hydrocephalus: historical trend. J Neurosurg Pediatr. 2013;11(2):170-177.

3. Vogel P, et al. Congenital hydrocephalus in genetically engineered mice. Vet Pathol. 2012;49(1):166-181.

4. Ohata S, et al. Loss of Dishevelleds disrupts planar polarity in ependymal motile cilia and results in hydrocephalus. Neuron. 2014;83(3):558-571.

5. Wang X, et al. SNX27 Deletion causes hydrocephalus by impairing ependymal cell differentiation and ciliogenesis. $J$ Neurosci. 2016;36(50):12586-12597.

6. Liu M, et al. Ulk4 is essential for ciliogenesis and CSF flow. J Neurosci. 2016;36(29):7589-7600.

7. Abdelhamed Z, et al. A mutation in $C c d c 39$ causes neonatal hydrocephalus with abnormal motile cilia development in mice. Development. 2018;145(1):dev154500.

8. Lim DA, Tramontin AD, Trevejo JM, Herrera DG, García-Verdugo JM, Alvarez-Buylla A. Noggin antagonizes BMP signaling to create a niche for adult neurogenesis. Neuron. 2000;28(3):713-726.

9. Sawamoto K, et al. New neurons follow the flow of cerebrospinal fluid in the adult brain. Science. 2006;311(5761):629-632.

10. Del Bigio MR. Ependymal cells: biology and pathology. Acta Neuropathol. 2010;119(1):55-73.

11. Breunig JJ, Arellano JI, Rakic P. Cilia in the brain: going with the flow. Nat Neurosci. 2010;13(6):654-655.

12. De Stefano ME, Herrero MT. The multifaceted role of metalloproteinases in physiological and pathological conditions in embryonic and adult brains. Prog Neurobiol. 2017;155:36-56

13. Zhang S, Chen D, Huang C, Bao J, Wang Z. Expression of HGF, MMP-9 and TGF- $\beta 1$ in the CSF and cerebral tissue of adult rats with hydrocephalus. Int J Neurosci. 2013;123(6):392-399.

14. Xu H, Xu B, Wang Z, Tan G, Shen S. Inhibition of Wnt/ $\beta$-catenin signal is alleviated reactive gliosis in rats with hydrocephalus. Childs Nerv Syst. 2015;31(2):227-234.

15. Barbolina MV, Stack MS. Membrane type 1-matrix metalloproteinase: substrate diversity in pericellular proteolysis. Semin Cell Dev Biol. 2008;19(1):24-33.

16. Zhou Z, et al. Impaired endochondral ossification and angiogenesis in mice deficient in membrane-type matrix metalloproteinase I. Proc Natl Acad Sci USA. 2000;97(8):4052-4057.

17. Jin G, et al. MT1-MMP cleaves D111 to negatively regulate Notch signalling to maintain normal B-cell development. $E M B O J$. 
2011;30(11):2281-2293.

18. Chan KM, et al. MT1-MMP inactivates ADAM9 to regulate FGFR2 signaling and calvarial osteogenesis. Dev Cell. 2012;22(6):1176-1190

19. Nishida C, et al. MT1-MMP plays a critical role in hematopoiesis by regulating HIF-mediated chemokine/cytokine gene transcription within niche cells. Blood. 2012;119(23):5405-5416.

20. Tang Y, et al. MT1-MMP-dependent control of skeletal stem cell commitment via a $\beta 1$-integrin/YAP/TAZ signaling axis. Dev Cell. 2013;25(4):402-416.

21. Wong HL, Jin G, Cao R, Zhang S, Cao Y, Zhou Z. MT1-MMP sheds LYVE-1 on lymphatic endothelial cells and suppresses VEGF-C production to inhibit lymphangiogenesis. Nat Commun. 2016;7:10824.

22. de Lucas AG, et al. Targeting MT1-MMP as an ImmunoPET-based strategy for imaging gliomas. PLoS ONE. 2016;11(7):e0158634.

23. Hambardzumyan D, Gutmann DH, Kettenmann H. The role of microglia and macrophages in glioma maintenance and progression. Nat Neurosci. 2016;19(1):20-27.

24. Hatoum A, Mohammed R, Zakieh O. The unique invasiveness of glioblastoma and possible drug targets on extracellular matrix. Cancer Manag Res. 2019;11:1843-1855.

25. Cinalli G, Spennato P, Cianciulli E, d'Armiento M. Hydrocephalus and Aqueductal Stenosis. In: Cinalli G, Sainte-Rose C, Maixner WJ, eds. Pediatric Hydrocephalus. Milan, Italy: Springer, Milano; 2019:279-293.

26. Feldner A, et al. Loss of Mpdz impairs ependymal cell integrity leading to perinatal-onset hydrocephalus in mice. EMBO molecular medicine. 2017;9(7):890-905.

27. Qin S, Liu M, Niu W, Zhang CL. Dysregulation of Kruppel-like factor 4 during brain development leads to hydrocephalus in mice. Proc Natl Acad Sci USA. 2011;108(52):21117-21121.

28. Fujitani M, Sato R, Yamashita T. Loss of p73 in ependymal cells during the perinatal period leads to aqueductal stenosis. Sci Rep. 2017;7(1):12007.

29. Goto J, Tezuka T, Nakazawa T, Sagara H, Yamamoto T. Loss of Fyn tyrosine kinase on the C57BL/6 genetic background causes hydrocephalus with defects in oligodendrocyte development. Mol Cell Neurosci. 2008;38(2):203-212.

30. Tramontin AD, García-Verdugo JM, Lim DA, Alvarez-Buylla A. Postnatal development of radial glia and the ventricular zone (VZ): a continuum of the neural stem cell compartment. Cereb Cortex. 2003;13(6):580-587.

31. Spassky N, Merkle FT, Flames N, Tramontin AD, García-Verdugo JM, Alvarez-Buylla A. Adult ependymal cells are postmitotic and are derived from radial glial cells during embryogenesis. J Neurosci. 2005;25(1):10-18.

32. Brazel CY, et al. Sox2 expression defines a heterogeneous population of neurosphere-forming cells in the adult murine brain. Aging Cell. 2005;4(4):197-207.

33. Lechtreck KF, Delmotte P, Robinson ML, Sanderson MJ, Witman GB. Mutations in Hydin impair ciliary motility in mice. J Cell Biol. 2008;180(3):633-643.

34. Fliegauf M, Benzing T, Omran H. When cilia go bad: cilia defects and ciliopathies. Nat Rev Mol Cell Biol. 2007;8(11):880-893.

35. Bruni JE. Ependymal development, proliferation, and functions: a review. Microsc Res Tech. 1998;41(1):2-13.

36. Abouhamed M, et al. Myosin IXa regulates epithelial differentiation and its deficiency results in hydrocephalus. Mol Biol Cell. 2009;20(24):5074-5085

37. Lattke M, Magnutzki A, Walther P, Wirth T, Baumann B. Nuclear factor $\kappa B$ activation impairs ependymal ciliogenesis and links neuroinflammation to hydrocephalus formation. J Neurosci. 2012;32(34):11511-11523.

38. Boutin C, et al. A dual role for planar cell polarity genes in ciliated cells. Proc Natl Acad Sci USA. 2014;111(30):E3129-E3138.

39. McClenahan FK, Sharma H, Shan X, Eyermann C, Colognato H. Dystroglycan suppresses Notch to regulate stem cell niche structure and function in the developing postnatal subventricular zone. Dev Cell. 2016;38(5):548-566.

40. Mirzadeh Z, Han YG, Soriano-Navarro M, García-Verdugo JM, Alvarez-Buylla A. Cilia organize ependymal planar polarity. $J$ Neurosci. 2010;30(7):2600-2610.

41. Delgehyr N, et al. Ependymal cell differentiation, from monociliated to multiciliated cells. Methods Cell Biol. 2015;127:19-35

42. Lehtinen MK, et al. The cerebrospinal fluid provides a proliferative niche for neural progenitor cells. Neuron. 2011;69(5):893-905

43. Mirzadeh Z, Doetsch F, Sawamoto K, Wichterle H, Alvarez-Buylla A. The subventricular zone en-face: wholemount staining and ependymal flow. J Vis Exp. 2010;(39):1938.

44. Tissir F, et al. Lack of cadherins Celsr2 and Celsr3 impairs ependymal ciliogenesis, leading to fatal hydrocephalus. Nat Neurosci. 2010;13(6):700-707.

45. Lavado A, Oliver G. Six3 is required for ependymal cell maturation. Development. 2011;138(24):5291-5300.

46. Gonzalez-Cano L, et al. p73 is required for ependymal cell maturation and neurogenic SVZ cytoarchitecture. Dev Neurobiol. 2016;76(7):730-747.

47. Eguren M, et al. The APC/C cofactor Cdh1 prevents replicative stress and p53-dependent cell death in neural progenitors. Nat Commun. 2013;4:2880

48. Yu X, Ng CP, Habacher H, Roy S. Foxj1 transcription factors are master regulators of the motile ciliogenic program. Nat Genet. 2008;40(12):1445-1453.

49. Jacquet BV, et al. FoxJ1-dependent gene expression is required for differentiation of radial glia into ependymal cells and a subset of astrocytes in the postnatal brain. Development. 2009;136(23):4021-4031.

50. Morimoto M, Liu Z, Cheng HT, Winters N, Bader D, Kopan R. Canonical Notch signaling in the developing lung is required for determination of arterial smooth muscle cells and selection of Clara versus ciliated cell fate. J Cell Sci. 2010;123(Pt 2):213-224.

51. Marcet B, et al. Control of vertebrate multiciliogenesis by miR-449 through direct repression of the Delta/Notch pathway. Nat Cell Biol. 2011;13(6):693-699.

52. Stubbs JL, Vladar EK, Axelrod JD, Kintner C. Multicilin promotes centriole assembly and ciliogenesis during multiciliate cell differentiation. Nat Cell Biol. 2012;14(2):140-147.

53. Kyrousi C, et al. Mcidas and GemC1 are key regulators for the generation of multiciliated ependymal cells in the adult neurogenic niche. Development. 2015;142(21):3661-3674.

54. Di Curzio DL. Animal models of hydrocephalus. Open Journal of Modern Neurosurgery. 2018;8(1):57-71. 
55. Shim JW, et al. Hydrocephalus in a rat model of Meckel Gruber syndrome with a TMEM67 mutation. Sci Rep. 2019;9(1):1069. 56. Kahle KT, Kulkarni AV, Limbrick DD, Warf BC. Hydrocephalus in children. Lancet. 2016;387(10020):788-799.

57. Banizs B, et al. Dysfunctional cilia lead to altered ependyma and choroid plexus function, and result in the formation of hydrocephalus. Development. 2005;132(23):5329-5339.

58. Ibañez-Tallon I, et al. Dysfunction of axonemal dynein heavy chain Mdnah5 inhibits ependymal flow and reveals a novel mechanism for hydrocephalus formation. Hum Mol Genet. 2004;13(18):2133-2141.

59. Coyle RC, Latimer A, Jessen JR. Membrane-type 1 matrix metalloproteinase regulates cell migration during zebrafish gastrulation: evidence for an interaction with non-canonical Wnt signaling. Exp Cell Res. 2008;314(10):2150-2162.

60. Golubkov VS, et al. The Wnt/planar cell polarity protein-tyrosine kinase-7 (PTK7) is a highly efficient proteolytic target of membrane type-1 matrix metalloproteinase: implications in cancer and embryogenesis. J Biol Chem. 2010;285(46):35740-35749.

61. Tan FE, et al. Myb promotes centriole amplification and later steps of the multiciliogenesis program. Development. 2013;140(20):4277-4286.

62. Takano T, Rutka JT, Becker LE. Overexpression of nestin and vimentin in ependymal cells in hydrocephalus. Acta Neuropathol. 1996;92(1):90-97.

63. Caillat C, Fish A, Pefani DE, Taraviras S, Lygerou Z, Perrakis A. The structure of the GemC1 coiled coil and its interaction with the Geminin family of coiled-coil proteins. Acta Crystallogr D Biol Crystallogr. 2015;71(Pt 11):2278-2286.

64. Carlén M, et al. Forebrain ependymal cells are Notch-dependent and generate neuroblasts and astrocytes after stroke. Nat Neurosci. 2009;12(3):259-267.

65. Sakamoto T, Seiki M. Cytoplasmic tail of MT1-MMP regulates macrophage motility independently from its protease activity. Genes Cells. 2009;14(5):617-626.

66. Takagishi M, et al. Daple coordinates planar polarized microtubule dynamics in ependymal cells and contributes to hydrocephalus. Cell Rep. 2017;20(4):960-972. 